\title{
Improving Error Correction Stage and Expanding the Final Key using Dynamic Linear-feedback Shift Register in Sarg04 Protocol
}

\author{
Omar Sh. Mustafa*, Ali H. Yousif, Dana F. Abdulqadir \\ Department of Information System Engineering, Erbil Technical Engineering College, Erbil Polytechnic University, Erbil, Iraqi Kurdistan
}

\section{${ }^{*}$ Corresponding author: \\ Omer Sh. Mustafa \\ Department of Information \\ System Engineering, Erbil \\ Technical Engineering \\ College, Erbil Polytechnic \\ University, Erbil, Iraqi \\ Kurdistan. \\ E-mail: omer.nematullah@ \\ epu.edu.krd}

\author{
Received: 23 September \\ 2018 \\ Accepted: 01 March 2019 \\ Published: 10 April 2019 \\ DOI \\ 10.25156/ptj.v9n1y2019.pp1-6
}

\section{A B S TR A C T}

The mechanism of quantum mechanics is considered as the principal method of quantum key distribution for transmitting a cryptographic key between users in unconditionally secure communication. In this paper, simulation and enhancement of the performance of SARGO4 protocol have been done in terms of error correction stage using multiparity rather than single parity. In addition, the suitable length of subblock was selected depending on the values of quantum bit error rate and round number. Dynamic linear-feedback shift register circuits have been used to extend the final key. These circuits are dynamically generated by the final key. A unique linear circuit is created for each generated key.

Keywords: Linear-feedback shift register; Multiparity; Quantum cryptography; Quantum key distribution; SARG04; Stream cipher

\section{INTRODUCTION}

An ideal cipher needs to offer unconditional security of symmetric key protocols without difficulties of key distribution. For this reason, many current protocols use an asymmetric key cipher (such as RSA) to exchange a key which offers a better efficiency in terms of throughput (James, 2012). Conventionally, one-time pad provides perfect information security, but it has a big problem concerning the length of the random key which should be equal to the length of the plaintext (Jabbar and Ahmed, 2013).

Multiparity quantum key distribution (MQKD) is a key distribution protocol where the same key is distributed to different parties using quantum mechanism. In the jargon of digital cryptography, MQKD can refer as a conference key distribution protocol establishes a common key among a number of users forming a conference (Abudhahir et al., 2012).

Since the first protocol, proposed by Benette and Brassard in 1984 (BB84), the quantum cryptographic (QC) protocols have been studied widely in recent years. It is observed that most of the later QC protocols are variants of BB84. Many problems incurred during the practical implementation of BB84 protocol. SARG04 protocol was proposed in 2004 by Scarani et al. (Hitesh et al., 2014). Among many candidates, SARG04 provides robust performance for the weak coherent pulse implementation of QC (Minal and Nisha, 2015). The researchers used four states of BB84 with different information coding to build SARG04 protocol. The SARG04 protocol is not much different from the original BB84 protocol (Stucki et al., 2011). The main difference does not refer to the "quantum" part of the protocol; it refers to the "classical" procedure of key sifting, which goes after quantum transfer. Such an improvement allows increasing security against photon number splitting attack (Jabbar and Ahmed, 2013). The SARG04 protocol in practice has a higher key rate than the BB84 protocol (Cyril et al., 2005).

Many papers have investigated this subject, for example: Jabbar and Ahmed, 2013, proposed a new method for error correction using multiparity instead of single parity in BB84 protocol under different attacks types. Muneer and Khaled, 2017, presented a deterministic and efficient threeparty QKD protocol for establishing a secret key between two untrusted users with the assistance of a third party and use the parity bit of the entangled spins to assist in the preparation of the secret states by applying two controlled- 
NOT gates. Xiangyu et al., 2017, proposed an efficient rate-adaptive reconciliation protocol suitable for practical continuous-variable QKD (CV-QKD) systems with timevarying quantum channel. This protocol changes the code rate of multi-edge type low-density parity check (LDPC) codes. (Mario et al., 2017) Multi-edge LDPC codes with low code rates and long block lengths were proposed for $\mathrm{CV}-\mathrm{QKD}$, to extend the maximum reconciliation distance between the two remote parties. Hua-Lei et al., 2016, provided the exact relationship between the secure key rate and the bit error rate in a six-state SARG04 protocol.

In this paper, a new method for error correction is proposed using multiparity instead of single parity and expanding the final key using privacy amplification depending on dynamic linear-feedback shift register (LFSR).

\section{MATERIALS AND METHODS OF QKD}

\section{Selection of a Suitable Block Length}

The step of selection of a suitable block length proposed to speed up the error correction process using multiparity instead of single parity in SARG04 protocol.

After making sure there are no intrusions on the data, the sifted key division process takes place into subblocks. The length of the subblock is identical on both (sender and recipient) to ensure the correction process is successful. In addition, the length of each subblock will be dynamically chosen depending on the quantum bit error rate (QBER) and round number. The proposed of suitable block length selection shows that the block length is $5 \leq \mathrm{L} \leq \mathrm{n}$ where $\mathrm{n} \leq$ sifted key length. The reason behind choosing 5 bits as block length is the adoption of using multiparity rather than single parity. Obviously, multiparity cannot be applied (apply single and multiparity at the same time) when the subblock length (2 Bits). Therefore, a 5-bit size was chosen for the calculation of multiparity. The single parity is calculated from the locations $(5,3$, and 1$)$, while the double parity is calculated from the locations ( 2 and 4 ). This means guessing the key when exchange the parities between sender and recipient on the intruder is so difficult. In this proposed, we apply the bisection search algorithm to find the error after exchange the parities between sender and recipient. This requires many rounds; in each round, a new block length is applied.

The formula used in this simulation to calculate the subblock length is given by: (Jabbar and Ahmed, 2013).

$$
\text { SubBlock Length }=\left(\frac{1}{\mathrm{QBER}}\right) *\left(0.3 * \mathrm{j}^{\wedge .2}\right)
$$

Where, $\mathrm{j}$ represents the current round number.
After applying many cases of simulation, this formula gives a good result and corrects the errors in a few rounds. After applying 20 rounds on the sifted key, the sender and recipient could correct all errors in the key successfully. This formula is suitable and effective to calculate the subblock length.

\section{Privacy Amplification Stages}

At this stage, both users should calculate the safety parameter (SP) as an assurance to remove the information leaked during the error correction stage and to minimize the intruder information.

So the final key length will be:

Final key length $=1 / 4$ raw key - Revealing portion to estimate QBER - N of discarded bits during error correction - W - SP.

Where $\mathrm{S}$ is the SP and $\mathrm{W}$ is the estimated Intruder information about the raw key.

\section{Apply Stream Ciphering on OKD Protocols in Simulation Program}

Another step has been added to SARG04 protocol to further secure the key and to exploit the key as efficiently as possible. In this paper, the final key used to generate dynamic LFSR by depending on the final key to select shift registers. Although the LFSR changes dynamically as the key length changes. Moreover, the expansion of the key is not as big as in traditional cryptographic methods (e.g. a gigabyte key can be generated from a 96-bit key). A small expansion is used for several reasons, and the most important reason is to avoid knowing even a small part of the circuit that generates this key. Therefore, different parts of the output of the used circuit were taken with the shifting. The size of the generated key in QKD is often relatively large. As a result, the LFSR will be very large and this will cause the problem of saving space for key storage and maintain a key to this size. Consequently, QBER is used to determine the amount of expansion. Whenever the amount of error is small, the amount of expansion will be large according to the following proposed relations:

If $(\mathrm{QBER} \leq 0.03)$ then

$$
\text { Call LFSR (Final Key, 15) }
$$

Else if $(\mathrm{QBER}>0.03)$ and $(\mathrm{QBER} \leq 0.06)$ then

$$
\text { Call LFSR (Final Key, 12) }
$$

Else if $(\mathrm{QBER}>0.06)$ and $(\mathrm{QBER} \leq 0.09)$ then

$$
\text { Call LFSR (Final Key, 9) }
$$

Else

$$
\text { Call LFSR (Final Key, 6) }
$$

end 
Where the numbers $(6,9,12$, and 15$)$ represent the amount of expansion, meaning that the key will expand to 6 times, for example, if the error rate is $>9 \%$.

\section{Generating Dynamic LFSR Circuits}

Let's assume the final key after applying all phases of (QKD) protocols was [100110010101].

The proposed algorithm, which generates dynamic LFSR, assumes that the final key is polynomial, then the algorithm will convert these bits to pseudo-random expanding form.

Hence, the above final key will be converted to the following form:

$$
\mathrm{X}^{11}+\mathrm{X}^{8}+\mathrm{X}^{7}+\mathrm{X}^{4}+\mathrm{X}^{2}+1
$$

The circuit is shown in Figure 1. The proposed method will generate the circuit and the key will expand according to the generated circuit. Moreover, there will be a unique and new different circuit for each key.

\section{MODEL ASSUMPTIONS}

The simulation design of SARG04 protocol is based on Figure 2, which summarizes all the possibilities of the four cases which can be sent:

From Figure 2, we conclude the following:

The probability of detecting sent state in column (1) is P1 $=0$ because both cases $\{(\uparrow, \searrow\})$ and $(\uparrow, \nearrow\})\}$ have been neglected. The probability of detecting sent to state in column (2) is P2 $=1 / 2$ because two out of four cases were detected. The probability of detection in column (3) is P3 $=0$. The probability of detection in column (4) and (5) is $\mathrm{P} 4=1 / 2$ and $\mathrm{P} 6=0$. The probability of detection in column (7) is $\mathrm{P} 7=1 / 2$. Finally, the probability of detection in column (8) is P8 $=0$. Thus, the overall probability of detection is as follows:

$$
\begin{array}{r}
\mathrm{P}_{\text {Total }}=\frac{1}{8} *\left(\mathrm{P}_{1}+\mathrm{P}_{2}+\mathrm{P}_{3}+\mathrm{P}_{4}+\mathrm{P}_{5}+\mathrm{P}_{6}+\mathrm{P}_{7}+\mathrm{P}_{8}\right) \\
=\frac{1}{8} *\left(0+\frac{1}{2}+0+\frac{1}{2}+\frac{1}{2}+0+\frac{1}{2}+0\right)=\frac{2}{8}
\end{array}
$$

Since only two cases can be detected from 8 cases, which represent $2 / 8$ from the whole cases. This means that the length of the key after the sifting process is $1 / 4$ of the length of the transmitted key.

\section{RESULTS}

\section{Simulation Results of SARG04 Protocol}

This protocol is simulated according to the following assumptions:

1. Four types of channels (perfect channel, low noise channel, medium noise channel, and high noise channel) were applied on SARG04 protocol by assuming that the channels are lossless.

2. There is no intrusion.

3. There are no rules to be exchanged between the two parties.

4. In the sifting stage, the four quantum states are declared.

5. Raw key length (5000-40000) bits.

\section{Stream Ciphering Simulation Results}

As previously mentioned, it is necessary to add another step of security (stream ciphering) to the SARG04 protocol to

\begin{tabular}{|c|c|c|c|c|c|c|c|c|c|c|c|c|c|c|c|c|c|c|c|c|c|c|c|c|}
\hline No. & \multirow{2}{*}{\multicolumn{2}{|c|}{$\frac{1}{0}$}} & \multicolumn{4}{|c|}{2} & \multicolumn{2}{|c|}{3} & \multicolumn{4}{|c|}{4} & \multicolumn{4}{|c|}{5} & \multicolumn{2}{|c|}{6} & \multicolumn{4}{|c|}{7} & \multicolumn{2}{|c|}{8} \\
\hline Sender's Random Bits & & & \multicolumn{4}{|c|}{$\mathbf{0}$} & & 1 & \multicolumn{4}{|c|}{1} & \multicolumn{4}{|c|}{1} & & 1 & \multicolumn{4}{|c|}{$\mathbf{0}$} & \multicolumn{2}{|c|}{0} \\
\hline Sender's Random Basis & \multicolumn{2}{|c|}{+} & \multicolumn{4}{|c|}{+} & & + & \multicolumn{4}{|c|}{+} & \multicolumn{4}{|c|}{$x$} & \multicolumn{2}{|c|}{$x$} & \multicolumn{4}{|c|}{$\times$} & \multicolumn{2}{|c|}{$\times$} \\
\hline Sender's States & \multicolumn{2}{|c|}{$\uparrow$} & \multicolumn{4}{|c|}{$\uparrow$} & & $\rightarrow$ & \multicolumn{4}{|c|}{$\rightarrow$} & \multicolumn{4}{|c|}{ 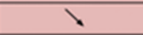 } & \multicolumn{2}{|r|}{$\searrow$} & \multicolumn{4}{|c|}{$\zeta$} & \multicolumn{2}{|c|}{$\nearrow$} \\
\hline Receiver's Random Basis & \multicolumn{2}{|c|}{+} & \multicolumn{4}{|c|}{$x$} & & + & \multicolumn{4}{|c|}{$x$} & \multicolumn{4}{|c|}{+} & & $x$ & & t & $t$ & & $>$ & K \\
\hline Receiver's Possible Measurement & 1 & & y & 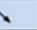 & 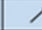 & & & $\rightarrow$ & $\mathrm{r}$ & 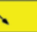 & > & $\zeta$ & & $\uparrow$ & - & $\rightarrow$ & & 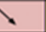 & & $\uparrow$ & $\rightarrow$ & $\rightarrow$ & 1 & 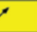 \\
\hline Receiver's Result & (c) & & 1 & 1 & 0 & 0 & & 1 & 1 & 1 & 0 & 0 & & 0 & 1 & 1 & & 1 & & 0 & 1 & 1 & $\mathrm{c}$ & j \\
\hline Sender Announcement states & $\begin{array}{l}\uparrow \\
\downarrow\end{array}$ & $\begin{array}{l}\uparrow \\
\dot{\gamma}\end{array}$ & $\begin{array}{c}\uparrow \\
\searrow\end{array}$ & $\begin{array}{l}1 \\
\vdots\end{array}$ & $\begin{array}{c}\uparrow \\
\searrow\end{array}$ & $\begin{array}{l}\uparrow \\
\vdots\end{array}$ & $\vec{v}$ & $\dot{\prime}$ & $\vec{v}$ & $\dot{\prime}$ & $\vec{\vdots}$ & $\dot{\prime}$ & $\begin{array}{l}\uparrow \\
\searrow\end{array}$ & 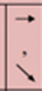 & $\begin{array}{c}\uparrow \\
\searrow\end{array}$ & $\dot{v}$ & $\dot{\imath}$ & $\vec{v}$ & $\begin{array}{l}\uparrow \\
\dot{y} \\
\end{array}$ & $\dot{\prime}$ & $\begin{array}{c}\uparrow \\
\dot{\gamma}\end{array}$ & $\vec{\prime}$ & $\begin{array}{l}\uparrow \\
\dot{y} \\
\end{array}$ & $\dot{\prime}$ \\
\hline Discovered States & & & & $\uparrow$ & $\uparrow$ & & & & & $\rightarrow$ & $\rightarrow$ & & & 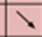 & 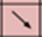 & & & & & $\zeta$ & $\zeta$ & & & \\
\hline The sifted Key & & & & 0 & 0 & & & & & 1 & 1 & & & 1 & 1 & & & & & 0 & 0 & & & \\
\hline $\begin{array}{l}\text { Correct } \\
\text { Error } \\
\text { Discard }\end{array}$ & 艿 & $\begin{array}{l}\text { 马ु్ } \\
\text { o. }\end{array}$ & $\begin{array}{l}\text { 렬 } \\
\text { 参 }\end{array}$ & 造 & 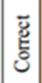 & $\begin{array}{l}\text { 己ृ } \\
\text { ğ }\end{array}$ & $\begin{array}{l}\text { 己ु } \\
\text { 品 }\end{array}$ & $\begin{array}{l}\text { 马ु్ } \\
\text { 㔛 }\end{array}$ & 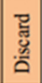 & हू & 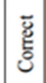 & 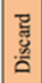 & $\begin{array}{l}\text { 马ुّ } \\
\text { o. }\end{array}$ & 氠 & 芯 & 胥 & 㞯 & 㞯 & $\begin{array}{l}\text { 范 } \\
\text { 参 }\end{array}$ & 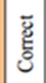 & "్. & 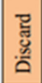 & 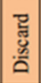 & 胥 \\
\hline
\end{tabular}

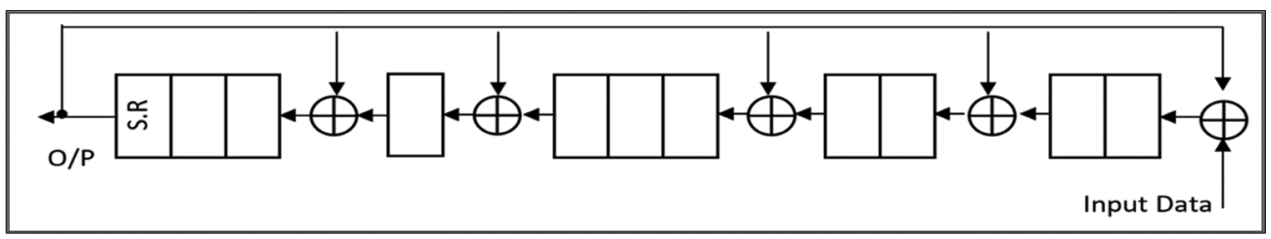

Figure 1: The dynamic linear-feedback shift register circuit generated from the above example

Figure 2: All SARG04 cases that can be sent by the sender and measured by the recipient using a random basis 


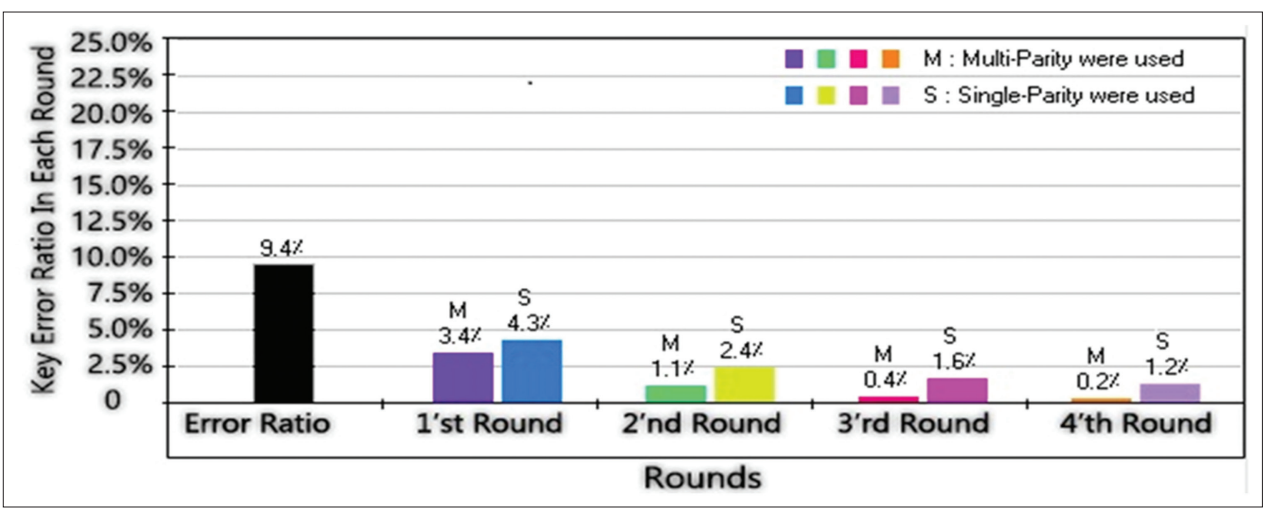

Figure 3: A comparison between the single parity and multiparity codes for the first four rounds of simulation (raw key = 20000 bits)

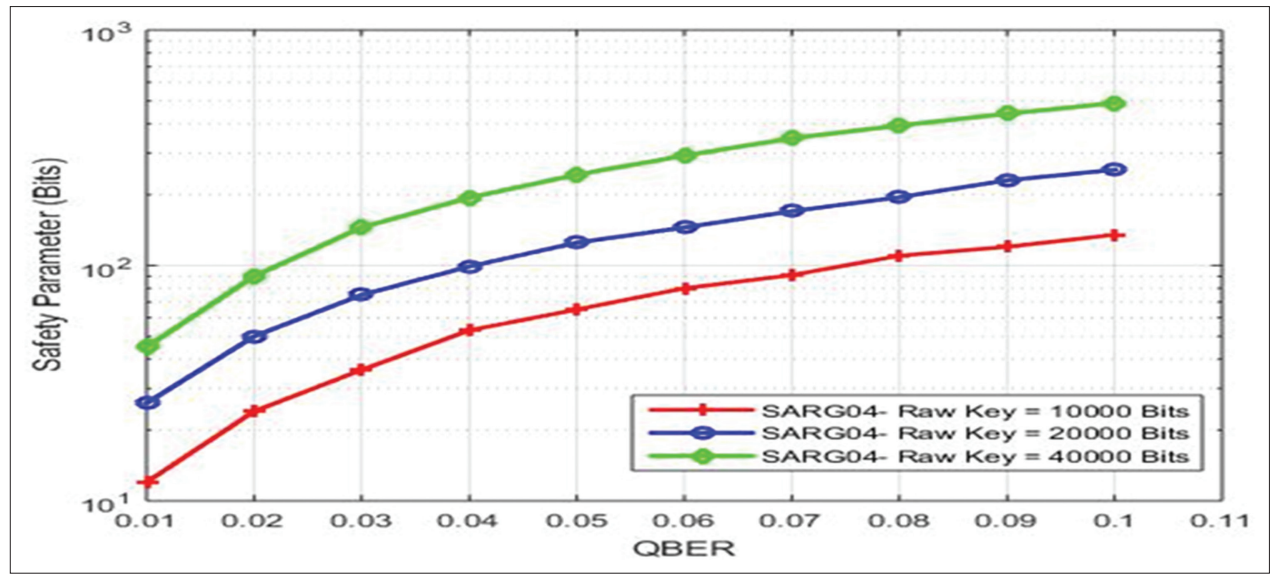

Figure 4: The relationship between safety parameter and quantum bit error rate for different raw key lengths

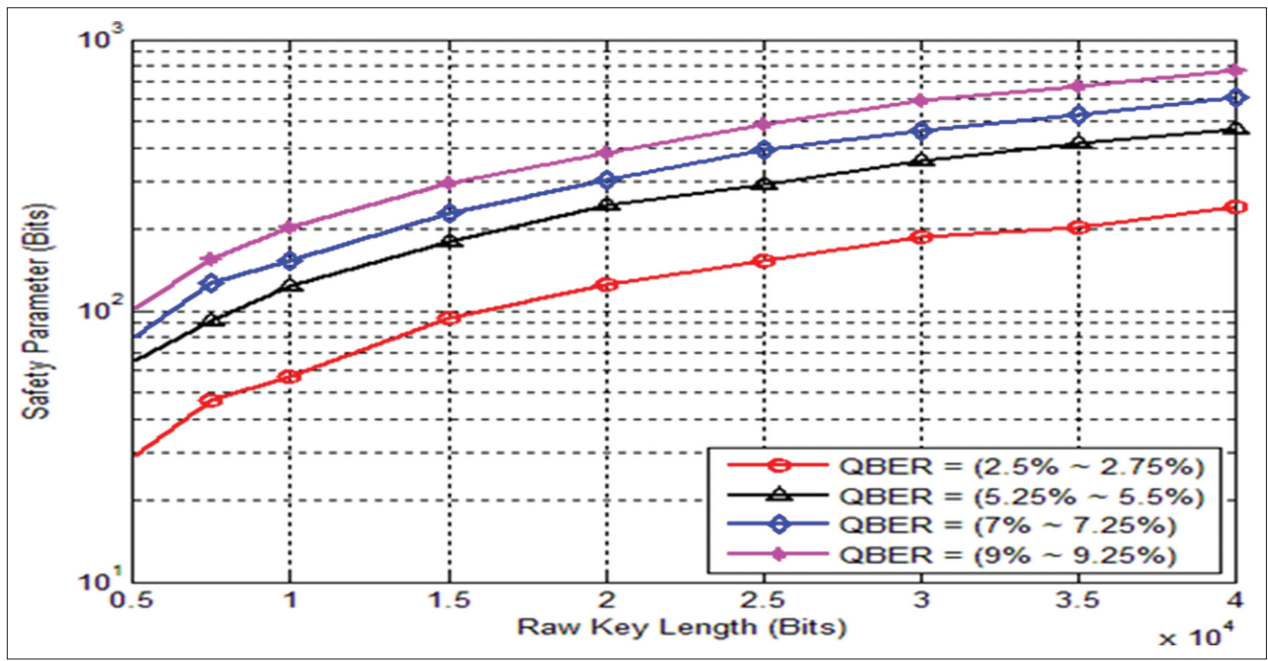

Figure 5: The safety parameter changes according to key size

expand the final key. The sender can use the expanded key to encrypt his messages. Figure 9 shows the generated polynomial equation that is used to generate dynamic LFSR. The figure also indicates that the final key length before expanding is 641 bits, but after applying stream ciphering expanded to 3846 bits. The expansion was 6 times while the polynomial can expand the key much more, but it could expose the used circuit (LFSR) to be disclosed by an attacker (Figures 3-8).

\section{CONCLUSION}

In this paper, the SARG04 protocol has been simulated, and some improvements were added to the SARG04 protocol at the error correction stage. Multiparity is used instead of single parity with some modifications to calculate the subblock length to be $\geq 5$ bits. Furthermore, the simulation 


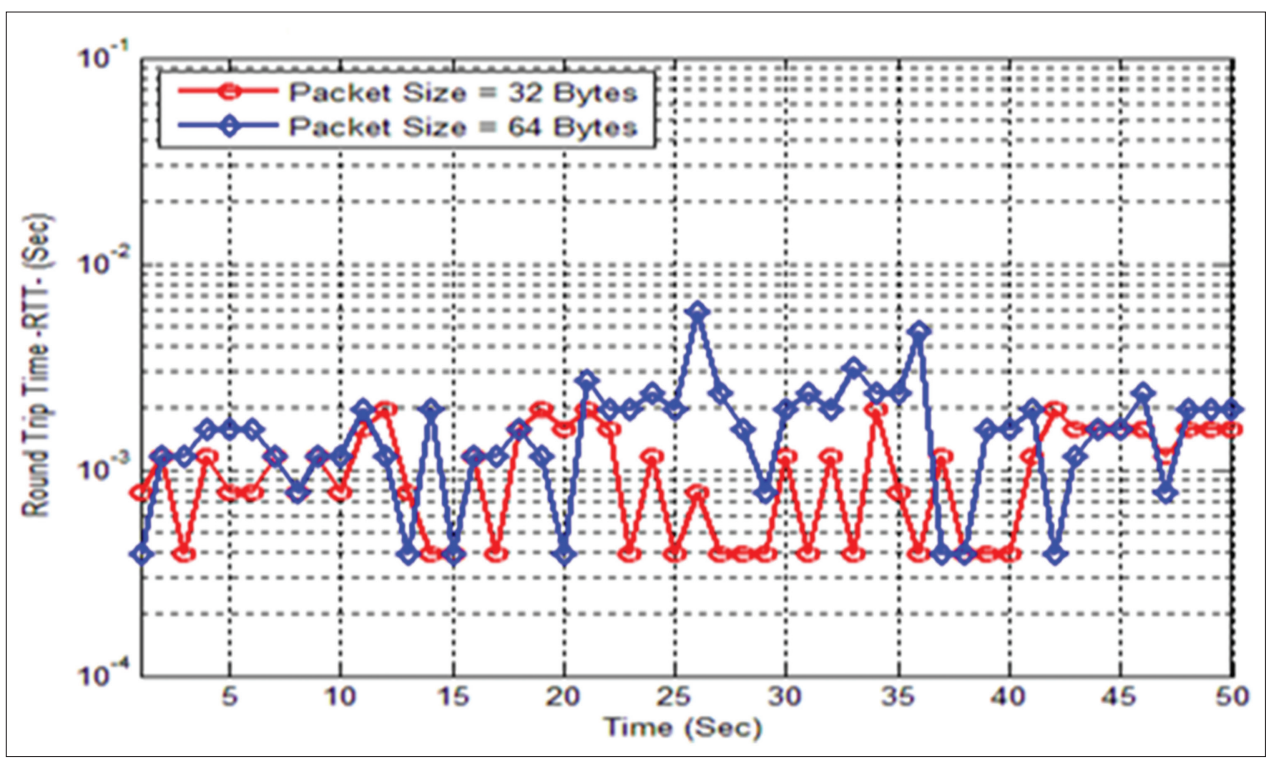

Figure 6: The calculation of round trip time in simulation software

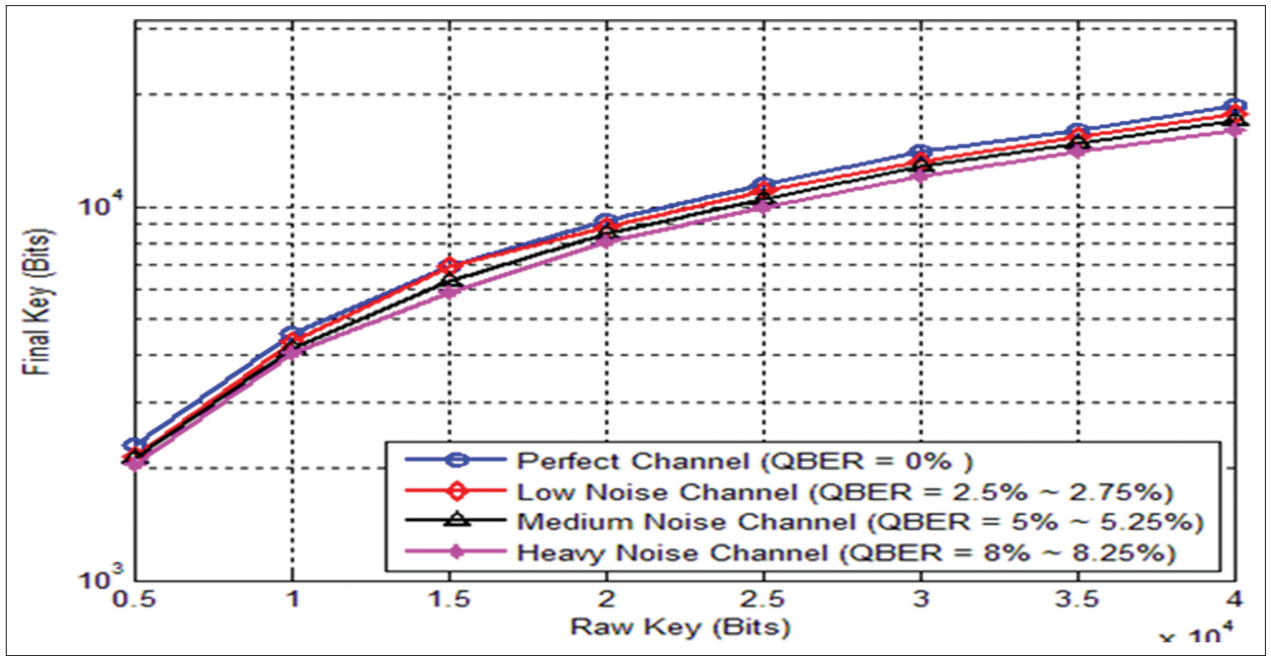

Figure 7: The final key length as a function of the length of the raw key and four channel types

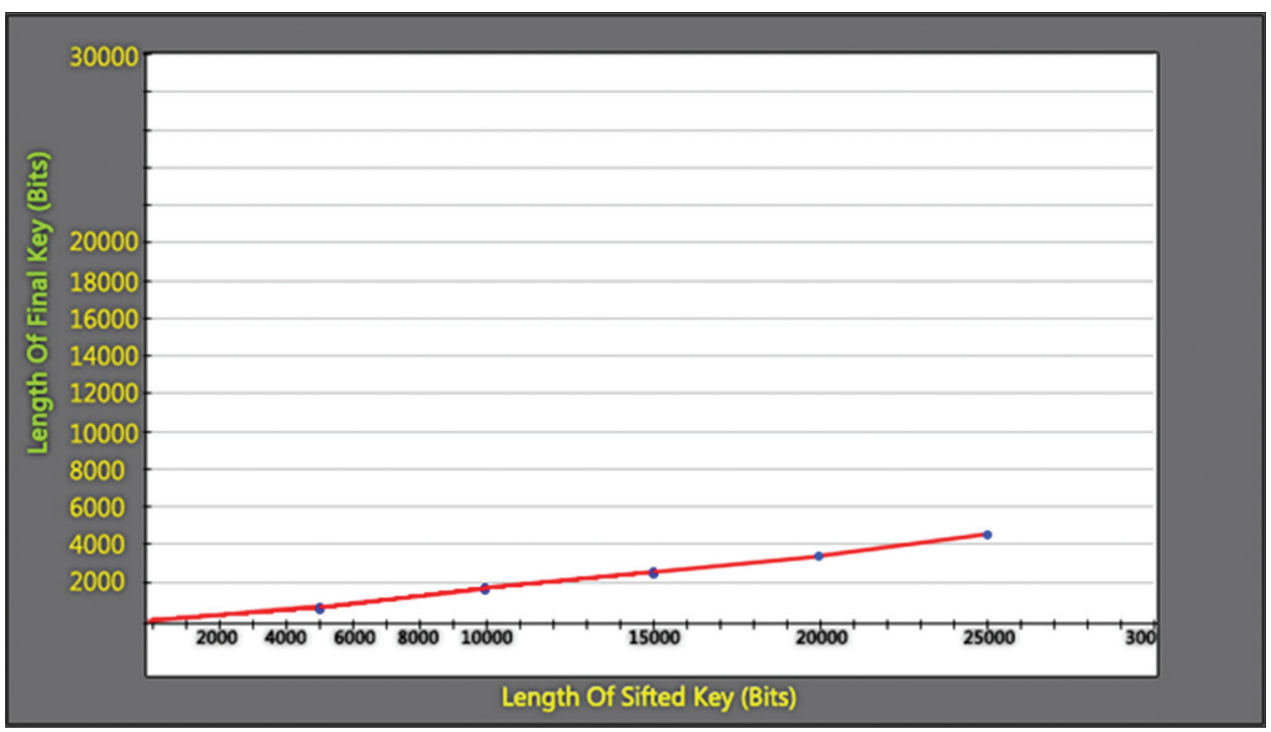

Figure 8: The final key length in comparison with the length of the sifted key 


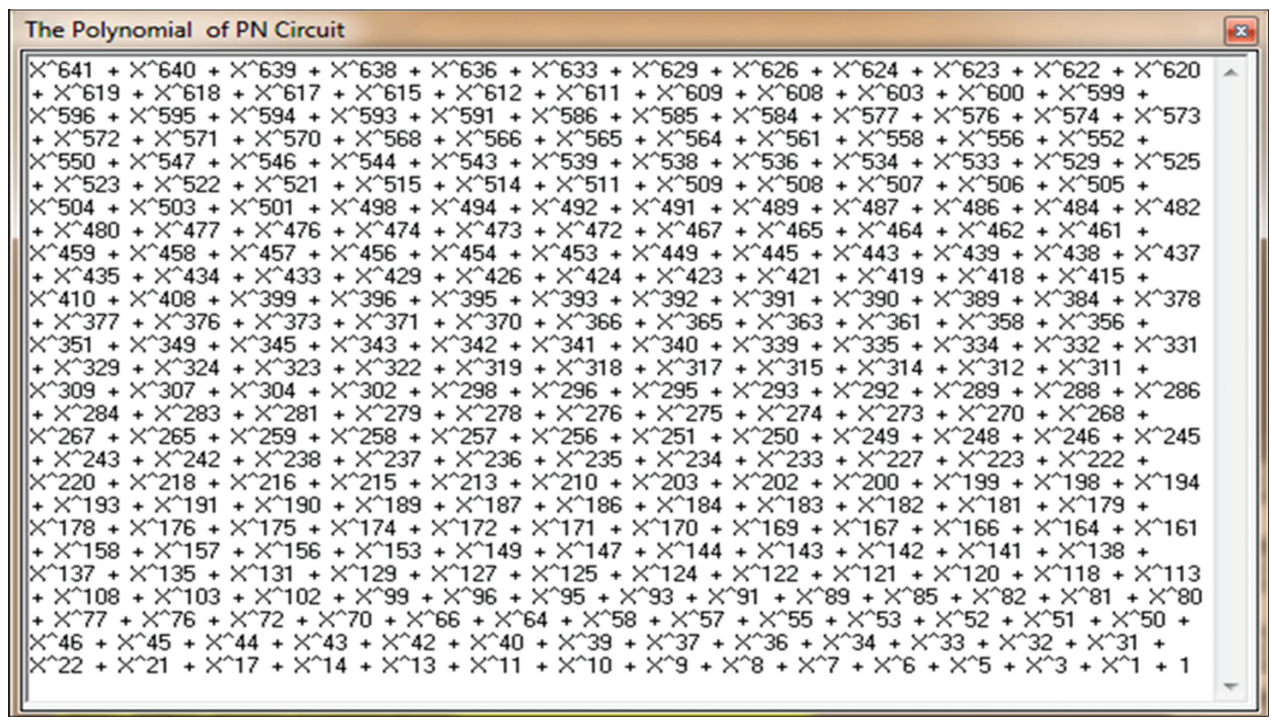

Figure 9: The polynomial of generating the linear-feedback shift register circuit which dynamically calculated

results showed that the execution of the process for 20 times makes all the errors correction in the key possible. It has also been shown that the error correction stage may leak some information to the intruder which can be avoided during the SP stage. This stage makes the algorithms that correct the error tend to perform better. The other optimization of the SARG04 protocol is the key expansion where the final key length has been increased for optimum length. The key distribution systems use a one-time pad to encrypt messages and need a long key. Therefore, improved stream ciphering was used in this paper to apply key expansion. It will use the final key to generate the pseudo-random expanding circuit dynamically (there will be a new expansion circuit for each generated key). The expansion will be restricted to QBER to prevent an intruder from detecting the expansion circuit. The higher QBER leads to lower expansion and vice versa.

\section{REFERENCES}

Abudhahir, B., Zukarnain, Z.A. and Subramaniam, S.K., 2012. Simple post quantum scheme for higher key rate multiparty quantum key distribution. International Journal of Network Security and Its Applications (IJNSA), 4(5). Available at: http://www.airccse. org/journal/nsa/0912nsa01.pdf. [Last accessed on 2017 Sep 1].

Cyril, B., Gisin, N., Kraus, B. and Scarani, V., 2005. Security of two quantum cryptography protocols using the same four qubit states. Physical Review, 2. Available at: http://www.cms.unige.ch/gap/ quantum/wiki/_media/publications: bib:physreva_72_032301. pdf. [Last accessed on 2017 Jan 1].

Hitesh, S., Gupta, D.L. and Singh, A.K., 2014. Quantum key distribution protocols: A review. IOSR Journal of Computer Engineering, 16(2), pp. 1-9. Available at: http://www.iosrjournals. org/iosr-jce/papers/Vol16-issue2/Version-11/A0162110109.pdf. [Last accessed on 2017 Mar 1].
Hua-Lei, Y., Fu, Y. and Mao, Y., 2016. Security of quantum key distribution with multiphoton components. Quantum Physics, 1. Available at: https://www.arxiv.org/pdf/1607.02366.pdf. [Last accessed on 2017 Apr 1].

Jabbar, A.I.A. and Ahmed, I.A., 2013. Applying multi-parity code to the quantum security protocol BB84 under different types of attacks. Al-Rafidain Engineering, 21(5), p.35. Available at: https://www. iasj.net/iasj?func=fulltext\&ald=79569. [Last accessed on 2017 Apr 1].

James, S.J., 2012. An Analysis of Error Reconciliation Protocols for Use in Quantum Key Distribution. M.S. Thesis, Air force Institute of Technology. Available at: http://www.dtic.mil/dtic/tr/fulltext/u2/ a557404.pdf. [Last accessed on 2017 Mar 1].

Mario, M., Feng, C., Zhang, L.M. and Gulak, G., 2017. Key reconciliation with low-density parity-check codes for longdistance quantum cryptography. Quantum Physics, 2. Available at: https://www.arxiv.org/pdf/1702.07740.pdf. [Last accessed on 2018 Jan 1].

Minal, L. and Nisha, S., 2015. On the Performance of Quantum Cryptographic Protocols SARG04 and KMB09. In: International Conference on Communication, Information and Computing Technology (ICCICT). Available at: http://www.ieeexplore.ieee. org/document/7045661. [Last accessed on 2017 Dec].

Muneer, A. and Khaled, M.E., 2017. Deterministic and efficient quantum key distribution using entanglement parity bits and ancillary qubits. IEEE Access, 5. pp. 25565-25575. Available at: http://www. ieeexplore. ieee.org/stamp/stamp.jsp?tp=\&arnumber=8101478. [Last accessed on 2018 Jan].

Stucki, D., Legré, M., Clausen, B., Felber, N. and Gisin N., 2011. Long term performance of the Swiss quantum key distribution network in a field environment. New Journal of Physics, 13. Available at: http://www.iopscience.iop.org/ article/10.1088/13672630/13/12/123001/meta. [Last accessed on 2017 May].

Xiangyu, W., Zhang, Y., Li, Z., Xu, B. and Yu, S., 2017. Efficient rateadaptive reconciliation for continuous-variable quantum key distribution. Quantum Physics, 1. Available at: https://www.arxiv. org/pdf/1703.04916.pdf. [Last accessed on 2018 Jan]. 